\title{
Future-proofing governance and BIM for owner operators in the UK
}

Ilias Krystallis BEng, BSC

BIM Lead, Rail, CH2M Hill Inc., London, UK

(corresponding author: ilias.krystallis@ch2m.com)

Vasileios Vernikos BEng, MSC

Head of BIM Development, CH2M Hill Inc., London, UK
Sam El-Jouzi BEng, CEng, MICE

Director of Rail Metros, CH2M, Hill Inc., London, UK

Pete Burchill BEng, CEng, MICE

Head of BIM Implementation, $\mathrm{CH} 2 \mathrm{M}$, Hill Inc., London, UK

Owner operators are managing and maintaining their infrastructure assets. In addition, depending on the national economic activity, they are being reactive or proactive in their response against uncertainty. Findings from this study showed that improvements can be achieved if the concept of future-proofing (FP) of assets - as a structured approach against uncertainty - becomes more explicitly defined. FP is the holistic process of taking security measures against uncertainty and being proactive throughout the organisation and its assets. In combination with information management, it ensures that asset management (AM) strategies will become responsive to a number of future changes in requirements. In this context, it is asserted that both FP and Building Information Modelling (BIM) suffer from a dearth of identification in the context of AM. Through a case study, this paper presents an approach that helps clients to future-proof AM at a strategic level. Furthermore, governance agendas for FP and BIM capabilities for future-proof information have been identified that owner operators and the supply chain can find useful.

\section{Introduction}

Future-proofing (FP) is discussed as a key issue in the pursuit of sustainable assets. Owners are seeking other ways to overcome the financial crisis by investing in the application of the sustainability agenda; and for an asset to achieve sustainability, the supply chain recognises that it has to be also future-proofed (Krygiel and Nies, 2008). Policymakers are defining what FP means for the good operation of services (DH, 2013). In this context, the Department of Health defined FP as strategic planning that responds to future changes in requirements, change of use, organisational strategic perspectives, national policy and changing climate. For infrastructure projects, Masood et al. (2013) defined FP as 'the process of incorporating future developments while changing from an unplanned and uncontrolled state to a planned and controlled state of a resilient infrastructure asset or product service system with minimal negative consequences'. For the purposes of this paper and summarising the above, FP can be defined as a proactive planning and management initiative and process employed by owners and the supply chain for mitigating risks found in asset management that acts as an urgent need against uncertainty. Ultimately, FP helps owners make better decisions during the asset life and creating ways to reduce the effects of problems arising in future events.

To investigate in detail how FP is applied within an enterprise, the authors observed a major infrastructure organisation. The observed organisation manages to allocate public funding through two main streams. The two streams operate as separate entities within the organisation but ensure that good communication exists between them. Among owner operators, there are in general two key categories of investment

n those delivered through capital projects

- those delivered by the operation and maintenance teams through their ongoing operations and maintenance (O\&M) processes.

The infrastructure asset management teams are responsible within the owner operator's portfolio management agendas for owning and maintaining their asset portfolio. The capital project schemes are generally focused on delivering large investment programmes for new sections of infrastructure, driven by the need for capacity enhancement. These schemes are typically implemented from the phases of inception to handover. On the other hand, O\&M schemes are delivered through a variety of mechanisms, such as a variety of contracts (lump sum or Design Build Finance and Operate (DBFO)) and also by a variety of stakeholders (joint ventures or house teams).

Considering the above, Building Information Modelling (BIM) should be the vehicle that progresses such discussion through its structured information management processes. Various governmental initiatives have been formed globally aiming to introduce BIM to the industry and promote guidance for all disciplines involved in building and infrastructure projects (Corenet, 2015; National Institute of Building Sciences, 2015; OpenBIM, 2013). In an information context, information FP can be defined as 'the process to ensure that required information is retrievable (reusable) throughout the whole life cycle of infrastructure assets or product service systems when needed' (Masood et al., 2013). 
Infrastructure Asset Management

Volume 3 Issue 1
Future-proofing governance and BIM for

owner operators in the UK

Krystallis, Vernikos, El-Jouzi and Burchill
The UK government is also promoting the BIM initiative (Cabinet Office, 2011). The perception is that cost efficiencies can be realised by use of a 'single collaborative source of truth' (BSI, 2007) during the design phase and through construction and commissioning and into its day-to-day operation where the end goal is the whole-life management of an asset (BSI, 2014).

The government's Construction 2025 strategy 2025 (HM Government, 2013) overarching target is to achieve a 'reduction in the initial cost of construction and the whole life cost of built assets by $33 \%$ '. To achieve this goal, the government plans are to ensure funding for key infrastructure projects of up to $£ 9 \cdot 5$ billion in rail, aviation and roads and reduced embodied carbon dioxide in infrastructure projects. The UK industry has successfully delivered some of the globe's largest infrastructure and regeneration projects (Black et al., 2015; Kershaw, 2012).

There are a number of different BIM definitions floating around. For the purposes of this study, the authors distinguish BIM as follows.

- $B I M$ (as a verb) is the process of generating and managing component data within an integrated database and parametric model throughout the project's design-build-operate life cycle.

- A Building Information Model (as a noun) is a digital representation of all physical and functional characteristics of a facility or site serving as a shared knowledge resource for information about the assets. This knowledge database forms a reliable basis for information exchange and decisions during a project's life cycle from inception onwards.

However, to break it down to the two primary features, BIM consists of the following.

- Geometry - Where is it? How long is it? How tall is it? What is it close to?

- Data - What is it called? What is it made of? Does it need any power? Does it have a warranty?

These form the core of what the government considers to be the BIM deliverable, with traditional documentation such as drawings and reports continuing to be delivered at the same juncture (Figure 1). With BIM there are now robust methodologies (BSI, $2007,2013)$ that articulate in detail the requirements and approaches for producing a project information model.

There is a trend within the construction industry that considers BIM to be a new 'autonomous and detached' solution for asset management (AM) (Pocock et al., 2014). This is primarily driven by the lack of existing asset data for many projects and a robust set of standards around data recording and delivery. In addition, there seems to be a lack of systematic guidance around FP and how much assets are change-ready to accommodate future changes. Considering the above, there needs to be a distinction made between the role of BIM and the mechanisms that ensure delivery of future-proof assets at pre-handover stages with respect to the life



Figure 1. Project outputs from a BIM project

cycle maintenance and management of assets. There then needs to be an understanding of the synergies between the two and how they can be managed to assist asset owner organisations.

\section{Methodology}

The chosen research methodology of this study was a case study. The investigated owner operator is a government-owned company that manages major linear infrastructure assets. The organisation operates in the UK and has more than 3500 employees. The study focused on investigating strategic decision-making aimed to address uncertainty in AM from the early stages. Combined data collection methods took place over a 3-month period, as they were deemed necessary to provide a deeper understanding of the research problem. A combination of collective instruments was adopted, and, thus, one-to-one interviews, telephone interviews, group interviews and processes review took place alongside a collection of archived documents that complemented the review process. The case study details are summarised in Figure 2. In the following sections, an overview of the observed challenges from the case study findings is presented.

\section{Results}

The FP approach includes the tools and processes to deliver futureproof projects by using BIM. The approach is conceptualised into three levels within an organisation.

Case study: government-owned owner operator operating major infrastructure projects

Research problem: strategic decision-making to address uncertainty in asset management from the early stages

Unit of analysis: an organisation's processes and IT systems ( 


Data sources: qualitative evidence



Figure 2. Case study overview 
Vision: The organisation defines what FP means for the business and informs the stakeholders and the supply chain about the vision of the organisation to satisfy FP goals as well as adoption of innovative technologies and processes.

- Strategy: The strategy involves the identifications of FP governance considerations across three agendas, government, strategy and information management agendas (Figure 3). Each agenda helps the business to explicitly define FP and information management and further refine in more details what both concepts mean within the business processes.

- Implementation: The last level covers how to apply FP and BIM strategies at the project level. It identifies the synergies of FP and BIM across a project's life cycle and showcases the strengths of using BIM in an FP framework.

Due to limitations, only the strategy level is covered in this paper.

\section{FP governance considerations per agenda}

To showcase how a mutation cycle (discussed below) can be initiated, the following emergent themes have been identified and are presented into two main categories, namely FP governance considerations across three agendas and the capabilities BIM can offer around FP solutions. For successful AM and delivery of future-proofed assets, a formal FP process needs to be formalised.
This process, depending on the agenda, varies in terms of what needs to be considered. The three high-level identified agendas are outlined here.

- Government agenda includes actions for both FP and BIM and works as a controlling and supporting mechanism that ensures clients and the supply chain are working together for the delivery of future-proof assets.

- Strategic management agenda includes decisions the clients are taking with the support of the supply chain. These decisions are about obtaining assets with the best possible change-readiness incorporated throughout their lifespan.

- Information management agenda includes processes and support from technology the supply chain needs to adopt in order to deliver the goals and aspirations set by their clients.

\section{Government agenda}

With regard to FP, there are two actions that could to be pushed further by the government.

- FP actions: These are actions that will allow the government to monitor and support the owner operators obtain futureproof assets. For this to be done, there is a need to foster organisations into creating mechanisms and processes that


Figure 3. FP governance considerations/responsibilities per agenda 
will support and enhance FP. The following actions have been identified.

Include contractual requirements that will further support the delivery of FP solutions.

- Establish an FP ranking system where each project/asset can be mapped against its FP capability on a national level.

- Support organisations into developing their FP key performance indicators (KPIs).

- BIM actions: The second action in this agenda should consider how to use BIM processes with regard to FP. The following actions have been identified.

- Many clients are now focusing on implementing their BIM standards and are starting to understand what BIM and its outputs could mean to them. There needs to be a central control by a government body that will ensure that these standards are sharing common principles. This can be proven to be highly effective for all asset owners regardless of sector as quality assurance standards and high-quality BIM services across all markets, sectors and projects will be ensured.

- Case studies that showcase evidence, lessons learnt and KPIs achieved from using BIM best practices for FP asset management.

- Guidance and support on how to approach BIM to ensure FP of information and what aspects of BIM can better support FP solutions and processes.

\section{Strategic management agenda}

On the delivery side, the clients need to work with their supply partners to agree over a common strategy on how change-ready assets should be delivered. The following decisions will support this goal.

- Strategic decisions: These are decisions that ensure that the clients' goals are clearly communicated to their suppliers. From the suppliers' side, there is a need to ensure that the clients' requirements are addressed. The following will support strategic decisions around FP.

- FP objectives are ingrained into a plan of works flow.

- The clients do not focus solely on capital cost but support the supply chain to deliver projects where design life is priority.

- The change-readiness framework (discussed below) is considered at the early stages of the project.

- Change of mind-set that considers 'payment by results' the only justifiable driver when discussions around future changes/upgrading emerge. Clients are starting to realise that upgrading of services/upskilling of resources is as important as the increase of customers' service provision.
- The clients should incentivise their suppliers to deliver projects where whole-life cost is reduced as opposed to solutions that are targeted for lower capital cost.

- Clear clarification of FP goals. For instance the clients should have clear understanding of where they want to have FP feed within their assets. It is uneconomical to have as a requirement an asset that should be $100 \%$ future-proofed, as this is unrealistic and can also be an expensive solution.

- Cooperative decisions: These types of decisions are supportive of the strategic decisions described earlier. These decisions highlight the need for a change in behaviour during decision-making. For example the delivery teams can adopt a more 'considerate' behaviour regarding the asset management teams. The following can support this.

- During design and construction delivery, if the teams identify that there are areas within the asset that could be considered to be 'problematic' in the near future, they should highlight this and try to find solutions that will overcome the issue rather than leaving it to be dealt by someone in the future.

- Decisions should be given by all stakeholders - that is, the teams that will eventually manage the assets should be invited at the early stages and share their knowledge. As this may raise conflicting interests, there is also a necessity that decisions should be based on pre-agreed weighted criteria.

- The supply chain should bring lessons learnt from other projects regarding the application of best practice in FP.

\section{Information management agenda}

Information management has now become an integral part of project delivery. BIM consists of processes around information management; furthermore, these are aligned with traditional project management processes. Furthermore, information management consists of processes that heavily rely on the support of technology.

- Implementation processes: To efficiently deliver data that can be used for FP, the following should be considered.

- The presence of Employer's Information Requirements (EIRs) as a contractual document that outlines information contractual deliverables is becoming more and more present in the contractual agreements for new projects. As part of the EIRs, there is a need for asset information needs to be clearly defined to ensure that asset data will be produced for efficient AM.

- During the maintenance cycle, EIRs may not be relevant, as in most cases design is not required. Therefore, only Asset Information Requirements need to be developed. These requirements identify which assets are maintained and are updated in the database. 
- As a response to the EIRs, the BIM Execution Plans (BEPs) need to ensure that the supply chain processes will deliver asset requirements that can be retrieved and reused by non-design experts.

- Following the BEPs, the project information models (PIMs) will include data that can be used by the AM teams to inform their operational decisions after handover.

- Technology support: To carry out the preceding processes, it is important to ensure that technology supports and not hinders these capabilities. The following are the minimum requirements.

- Interoperability should be ensured across BIM tools (BIM authoring tools, common data environment) and AM systems, software-agnostic solutions such COBie, .csv and IFC files could be used.

- By recognising that data will be maintained in an electronic format throughout an asset's lifespan, there is a need to ensure that each piece of data will have a unique ID so that it will be protected from being lost or overwritten.

- AM systems have the functionalities to receive and capture data that can be used for life cycle decisions.

\section{Change-readiness framework}

The following change-readiness framework provides clarifications regarding the range of 'what' could change as well as an indication of 'when' this could happen (Figure 4). Slaughter (2001) discussed three types of changes that can occur within an asset; these changes are regarding the asset's flow, function and capacity.

Flow: The first type of change involves changes in

- environmental flows - for example a change may be required to occur due to a climatic change or physical environmental conditions within the asset

- flow of people/things that may occur from an organisational change decision.

Function: For infrastructure projects, such changes may occur as result of

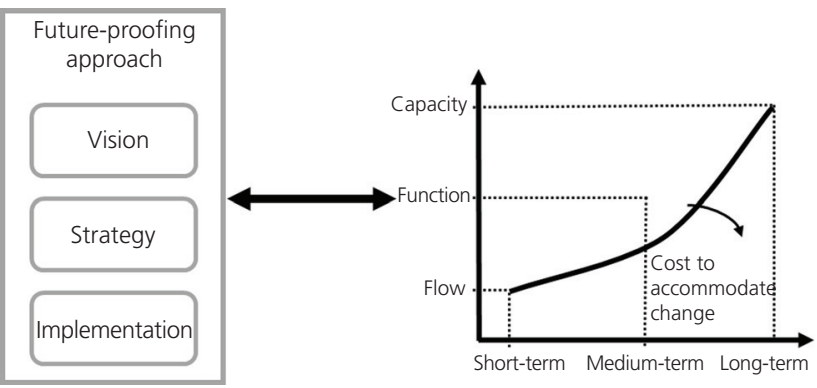

Figure 4. Change-readiness framework aligned with the FP approach
- reusing existing functions - upgrading an existing space for better performance

- creating new functions - creating an existing space for additional functions

- changing for different functions - altering the space for different functions to apply.

- Capacity: The third type of change is related to the structural transformation of the asset to meet specific performance requirements. Changes in capacity may occur from changes regarding the asset's 'volume' and/or 'loads'. Essentially, these changes are focusing on 'size'. Transformation is more rigid compared with the first two categories; these types of alterations are also more expensive.

De Neufville et al. (2008) categorised three types of applications as they emerge into an asset life cycle: operational, tactical and strategic applications. The asset will perform as it was originally designed, but, in addition, there can be an additional ability within the asset that can be described as a switch. In effect, the owner can switch this ability on and off depending on the internal and external factors that emerge. Each type of application can be considered as moving from one level to the next while directing the asset to adapt to changing needs dynamically.

- Operational or short-term applications are the lightest form of change and the easiest, as these can be applied on a daily or weekly basis. This change finds application in light systems. The application can be cost-effective while endorsing a rapid ongoing change in the short term.

- Tactical or mid-term applications reflect a more permanent response in the change scale and thus require significant capital to be reverted. In order for this application to be effective, the initial capital cost of the asset could be higher $(10-20 \%)$. The application is used to address medium-term uncertainty, and to become effective. it could take a few weeks of implementation.

- Strategic or long-term applications are strategies that owner operators could apply in considering the end life of the asset. The effort of deploying this option is to increase significantly the life expectancy of the asset. Owners would expect this application to become effective after years of handover of the asset.

Moreover, cost is increasing from one application to the next. The change-readiness framework is used for informing an effective FP approach. An exercise of identifying options across this graph reflecting the asset's lifespan can inform the 'lifespan asset optioneering' exercise in the mutation cycle as discussed in the following section.

\section{BIM capabilities that support FP}

As discussed in the previous sections, there is real opportunity for both the clients and the supply partners to benefit from the use of BIM. This metacritique serves as an identification of the qualitative competences that exist in a BIM process environment. 
The following supportive capabilities have emerged, and these are summarised in Figure 5.

- Flexible data: Flexibility in this instance means that the data can be used by multiple teams across the project and throughout the asset's lifespan for multiple purposes. To ensure data flexibility, there needs to be a standard procedure for information exchange and systems interoperability. All of these challenges are trying to be addressed within a BIM process.

- Optioneering capabilities: Optioneering studies are becoming more easily implemented within a BIM process framework. Information has become easily accessible, and this is further supported by technology advancements. BIM not only offers the possibility of running many 'what-if' scenarios to choose the best possible solution based on the clients' requirements but it can also be used for evaluating possibilities regarding the scenarios an asset can accommodate post-handover (life cycle asset optioneering).

- Project evaluation: BIM is also becoming a quality assurance process for evaluating models and validating data input. In terms of FP, BIM can be used for quantifying how futureproof an asset can be, identifying resilient-sensitive areas and informing decision-making.

- Standardised object catalogues: Just like in the automotive industry, it is now possible to have libraries of components that can be reused from project to project, saving time and resources. These components and their properties can in addition be backed up with evidence - that is, performance, durability, maintenance conditions and so on - to better support FP decisions.

- Whole-life costing: Essentially this is the main purpose of moving towards BIM process delivery. The PAS 1192-2 (BSI, 2013) and PAS 1192-3 (BSI, 2014) specifications outline how a capital expenditure model will be used to support operational expenditure purposes. With BIM it is now possible to have whole-life cost models in place and evaluate future scenarios about the asset in question. The design models can be linked to cost databases that effectively

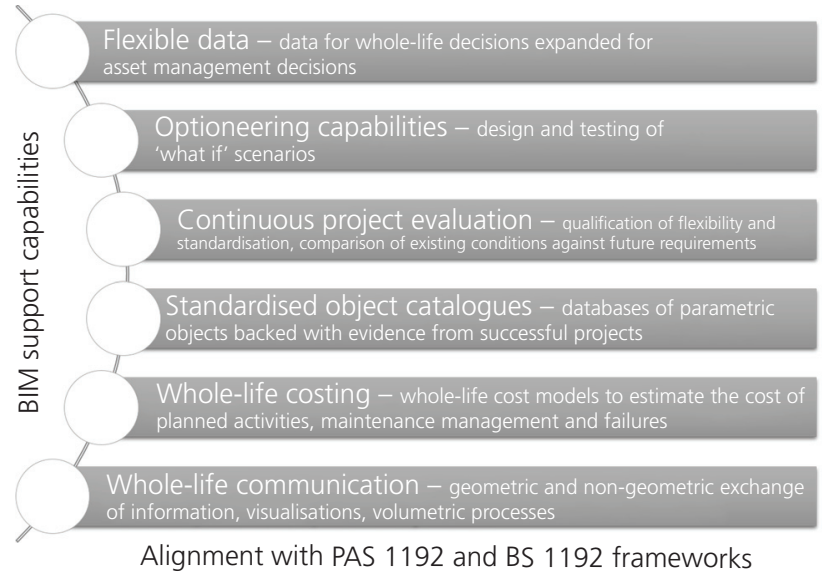

Figure 5. BIM support capabilities for FP development investigate the best solutions from a pool of solutions that are ingrained within the design model itself.

- Whole-life communication: Communication of requirements, rich-based complex databases and early data that can be useful for the AM teams is only one example that makes communication the strongest capability of BIM.

Communication is the key to the effective delivery of changeready assets, and volumetric design, software-agnostic exchange packages (i.e. COBie) and visualisations are some of the examples of this capability.

Life cycle asset optioneering and mutation asset life cycle The previous sections captured the governance considerations for life cycle asset optioneering as well as the qualities offered from the adoption of BIM. In this section, a mechanism is proposed that takes into effect the governance considerations (3 agendas) and BIM capabilities. Present approaches involve the client procuring for a new development suggesting that the supply teams need to provide a number of proposals (approximately three) and then, after evaluation of the three propositions, the team will produce a feasibility study based on a single preferred option (SPO). FP takes a different stance and suggests that the clients should steer their approaches on a different direction. By considering the above, it is then possible to re-engineer the procurement process and stimulate alternative design approaches that will protect the lifespan of the asset. Implying a switch is incorporated into the asset; it is then possible that during its lifespan the asset will be able to readjust itself into the business needs the organisation is required to respond. The asset lifespan in essence becomes the mutation cycle identified in Figure 6, and instead of an SPO, the clients acquire a current preferred option (CPO) solution. A CPO identified in the initial stages ensures that the design will have the insurance embedded into the asset that will allow it to address change at a set period in time.

Post-handover the asset lifespan starts, and the asset will eventually be challenged by many internal (new policies, change of services etc.) and external factors (political, environmental etc.). This is when the 'mutation' cycle initiates and FP comes in effect. Unlike the present planning approaches and an SPO outcome, the mutation cycle is divided into mutation periods and each period has a CPO (top rows in Figure 6). The asset then remains current to the new set of factors that determine the asset's use at a particular span or period in time. Assuming the factors will change at some point in the future, then the mutation period will come to an end and the asset will readjust itself. The organisation will re-evaluate its business scope and identify from a pool of scenarios the most suitable CPO.

The life cycle asset optioneering activity materialises from the context of the change-readiness framework and the BIM capabilities (see previous section). The outcomes - that is, the CPOs - are stored into the asset's database, where the clients and AM teams have access post-handover to inform their decisions. The CPOs consider a range of occurring changes during the asset's lifespan which are informed by the change-readiness 


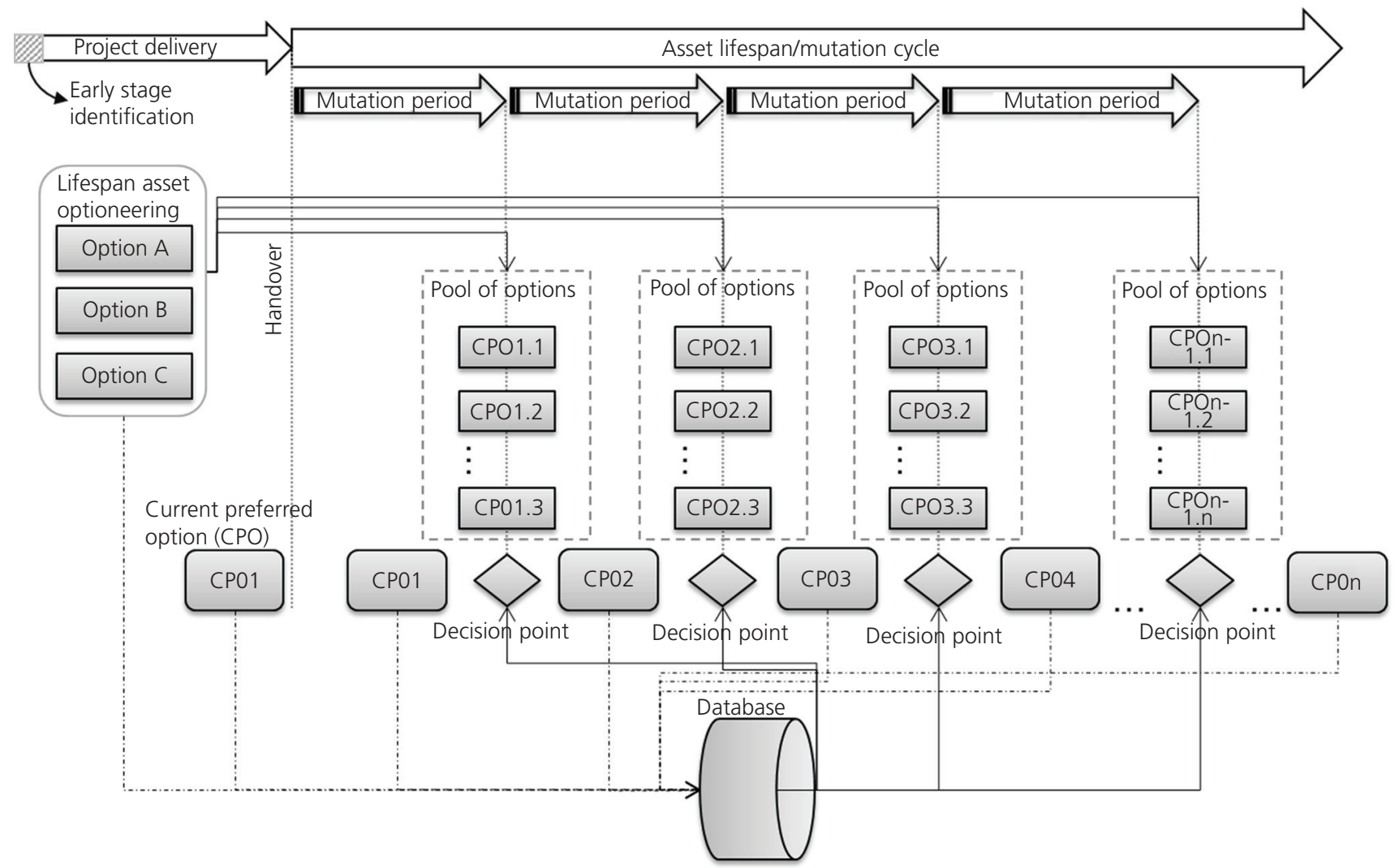

Figure 6. Life cycle asset optioneering and mutation asset life

cycle

framework (discussed earlier). In terms of data representation, these could be models, drawings, systems, components and attributes (Figure 7). The data will be stored in the BIM database, and at a later point in time, these will be available for retrieval and be used at any of the decision points shown in Figure 6 .

The CPOs with the use of the employed BIM capabilities and through AM data capture processes will feed information back to the database, increasing the volume of data and also updating existing data. It is outside of the scope of this study to describe in detail the process of retrieving and reusing information from

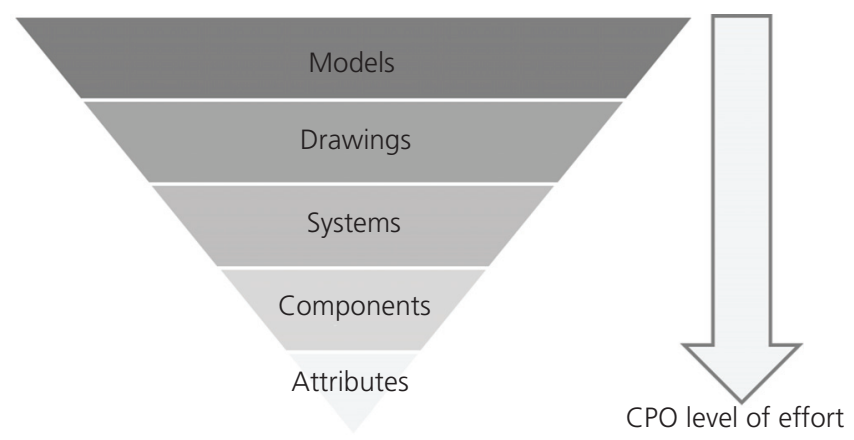

Figure 7. CPO levels of effort databases to inform decisions; however, extended research has been taken by other researchers (Demian, 2004; Masood et al., 2013). Lastly, the mutation cycle cannot be implemented if the above process is not identified by the delivery teams and the client during the early project stages of delivery.

\section{Discussion}

For efficient AM, clients and their supply partners should work together to establish an infrastructure of information delivery prior to the handover stage. The assessments that were carried for the purposes of this study showed that AM can be detached and not find support regarding the handover of data that are 'fit for purpose'. In this instance, data that are fit for purpose are those that are produced from inception to construction and can further be used for O\&M purposes post-handover within a BIM environment. AM frameworks (Taggart et al., 2014) can be effectively applied in infrastructure projects if there is (a) provision of useful information and $(b)$ awareness that there is uncertainty hidden within the asset's lifespan.

Clients should push their suppliers for more holistic outcomes and target for whole-life cost reductions within their schemes. Indeed the government mandate is that public projects should be delivered in BIM, but that does not warrant that the assets will still be fit for purpose 50 years after their completion. Nevertheless, FP 
Future-proofing governance and BIM for

owner operators in the UK

Krystallis, Vernikos, El-Jouzi and Burchill



Figure 8. The MacLeamy curve and FP impact on cost of design changes

should not only be about major expansions. Major savings can be accomplished by simply setting the right fit-for-purpose data in place and in an accessible format. This will mean that other teams will be able to use and make informed decisions regarding the life of the asset. These informed decisions will originate from the data that comes with the delivered physical asset.

Construction needs to drive towards a focus on whole-life cost reduction rather than capital cost reduction, and findings from Mevellec and Perry (2006) and Wang (2011), among others, note the importance of whole-life costing. Furthermore, it was highlighted that standardised solutions need to have embedded agility in their uses so that they can adapt. In addition, delivering projects that have the ability to deconstruct rather than being demolished is another important aspect of design, and a great example of such design approaches can be seen in King's Cross Station (King's Cross Central Limited Partnership, 2014).

The realisation that change is inevitable should question whether mandatory changes will occur too late when future requirements demand it - and thus having increased cost of changes - or ideally a 'platform of awareness' will be built in which change will occur as Hamel and Prahalad (1996) suggested, in a 'controllable environment'. This platform eventually should include a FP 'insurance' procedure and fit-for-purpose data as deliverables of this process. As shown in Figure 8, the cost of design changes can be reduced in the MacLeamy curve if a BIM workflow is implemented early in the project and, in addition, the project is covered by a future-proof insurance. In order to protect the assets, a series of governance measures and change in the optioneering process is suggested.

\section{Conclusions}

To future-proof assets and consequently their management there is a need for the establishment of a series of high-level protection measures against uncertainty. These measures have been outlined within three agendas, namely government, strategic management and, due to the opportunities that BIM brings, information management. These agendas can be implemented only if they are supported by the BIM capabilities offered within the BIM process. Both agendas and BIM capabilities should work as building blocks that support AM. If the AM teams have access to such information, they then have valuable support and it is then possible that decisions could be taken around future-proof life cycle development. The decisions will be better supported with evidence and will be more informed, hence leading to better life cycle decisions (Figure 9).

To achieve the above, the teams are working on a changereadiness framework to inform the life cycle asset optioneering process. The outcomes of this optioneering activity are used to inform decisions taken throughout the mutation cycle. The mutation cycle is essentially the asset lifespan amplified with FP to withhold against uncertainty, which essentially informs the AM teams to make better decisions. As cost reductions in the construction and the sustainability agendas become more and more important, both the clients and their supply partners aim throughout the life cycle of the asset to facilitate assets that are able to respond to a mutation cycle.

\section{REFERENCES}

Black M, Dodge C and Lawrence U (eds) (2015) Crossrail

Project: Infrastructure Design and Construction. Institution of Civil Engineers, London, UK.

BSI (2007) BS EN 1192:2007: Collaborative production of architectural, engineering and construction information - code of practice. BSI, London, UK.

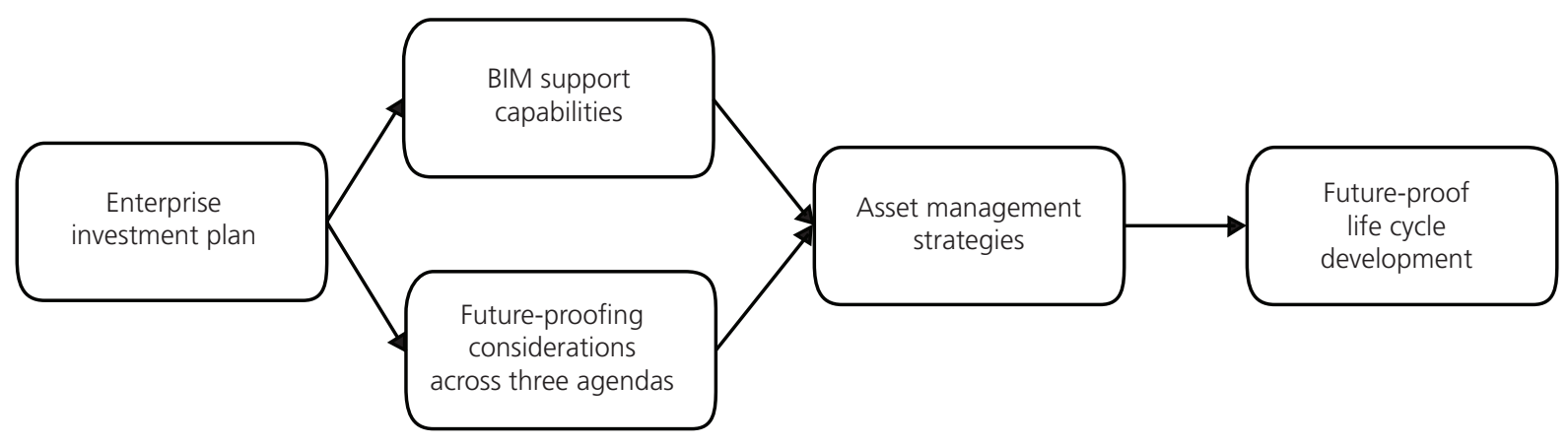

Figure 9. Building blocks to support future-proof AM decision 
BSI (2013) PAS 1192-2: Specification for information management for the capital/delivery phase of construction projects using building information modelling. BSI, London, UK.

BSI (2014) PAS 1192-3: Specification for information management for the operational phase of assets using building information modelling. BSI, London, UK.

Cabinet Office (2011) Government Construction Strategy. Cabinet Office, London, UK.

Corenet (2015) CORENET e-Information System, Corenet, Singapore. See https://www.corenet.gov.sg/general/e-info.aspx (accessed 05/04/2015).

de Neufville R, Lee YS and Scholtes S (2008) Using flexibility to improve value-for-money in hospital infrastructure investments. In 2008 First International Conference on Infrastructure Systems and Services: Building Networks for a Brighter Future (INFRA). IEEE, Piscataway, NJ, pp. 1-6.

Demian P (2004) CoMem: Design Knowledge Reuse from a Corporate Memory. PhD thesis, Stanford University, Stanford, CA, USA.

DH (Department of Health) (2013) HTM 07-07 - Sustainable Health and Social Care Buildings. DH, London, UK.

Hamel G and Prahalad CK (1996) Competing for the Future. Harvard Business Press, Boston, MA, USA.

HM Government (2013) Construction 2025 - Industrial Strategy Government and Industry in Partnership. Crown, London, UK

Kershaw S (2012) Delivering London 2012: transport programme assurance. Proceedings of the Institution of Civil Engineers Transport 165(4): 241-248, http://dx.doi.org/10.1680/tran.12. 00042.

King's Cross Central Limited Partnership (2014) Future-proofThe Infrastructure at King's Cross. King's Cross Central
Limited Partnership, London, UK. See http://www.kingscross. co.uk/infrastructure (accessed 10/06/2015).

Krygiel E and Nies B (2008) Green BIM: Successful Sustainable Design with Building Information Modeling. Sybex, Hoboken, NJ, USA.

Masood T, Cuthbert R, McFarlane DC and Parlikad AK (2013) Information futureproofing for large-scale infrastructure. IAM Asset Management Conference 2013, pp. 1-23.

Mevellec P and Perry N (2006) Whole life-cycle costs: a new approach. International Journal of Product Lifecycle Management 1(4): 400-414.

National Institute of Building Sciences (2015) National BIM Standard - United States - Version 2. National Institute of Building Sciences, Washington, DC, USA. See http://www. nibs.org/?page=standards (accessed 02/04/2015).

Open BIM (2013) Autodesk Supports BIM interoperability | \#IFC. Open BIM. See http://open.bimreal.com/bim/index.php/2013/ 11/29/autodesk-supports-bim-interoperability-ifc/ (accessed 02/ 04/2015)

Pocock D, Shetty N, Hayes A and Watts J (2014) Leveraging the relationship between BIM and asset management. Infrastructure Asset Management 1(1): 5-7.

Slaughter ES (2001) Design strategies to increase building flexibility. Building Research \& Information 29(3): 208-217.

Taggart A, Tachtsi L, Lugg M and Davies H (2014) UKRLG framework for highway infrastructure asset management. Infrastructure Asset Management 1(1): 10-19.

Wang N (2011) Multi-criteria decision-making model for whole life costing design. Structure and Infrastructure Engineering 7(6): $441-452$.

\section{WHAT DO YOU THINK?}

To discuss this paper, please submit up to 500 words to the editor at journals@ice.org.uk. Your contribution will be forwarded to the author(s) for a reply and, if considered appropriate by the editorial panel, will be published as a discussion in a future issue of the journal. 